\title{
No Demonstrable Relationship Was Found between Alcohol Dependence and Concomitant Drug Abuse amongst Detainees in Police Custody in West Yorkshire, England
}

\author{
Remy Bahl*, William P. Tormey \\ School of Biomedical Sciences, University of Ulster, Coleraine, UK \\ Email: remybahl@hotmail.com, billtormey@gmail.com
}

Received 22 January 2014; revised 23 February 2014; accepted 21 March 2014

Copyright (C) 2014 by authors and Scientific Research Publishing Inc.

This work is licensed under the Creative Commons Attribution International License (CC BY).

http://creativecommons.org/licenses/by/4.0/

(c) (i) Open Access

\begin{abstract}
The behavioural effects of alcohol and drug abuse may lead to arrest by the police. Individuals who abuse one substance may be at risk of developing multiple drug dependencies. Using the forensic records, data were collected on 50 successive subjects detained in police custodies across West Yorkshire, England who had gave history of alcohol addiction to a single practitioner. The degree of correlation between alcohol dependency and illicit drug usage was assessed by calculating the Spearman's Rank coefficient. Thirty three subjects in this study did not use any illicit drugs. There was no correlation between alcohol dependency and concomitant drug abuse in this group. Spearman's coefficient was statistically insignificant $(p=0.230)$. Kruskal's Gamma, which is used for comparing ordinal data, also failed to show a significant link between the alcohol and drug group $(p=0.185)$. As the degree of alcohol dependency increased, co-use of other drugs decreased. Conversely as the use of stronger drugs increased, co-use with alcohol and other drugs increased. Alcohol dependency is a distinct disorder. Once alcoholism had set in, the use of other drugs falls. The forensic behavioural patterns linked alcohol with "violent disorder" and Class A drug abuse with "organised crime". This study does not support the contention that most alcohol dependent individuals will also abuse illicit drugs.
\end{abstract}

\section{Keywords}

Alcohol, Drugs, Correlation

\footnotetext{
*Corresponding author.
} 


\section{Introduction}

In 2011 there were 8748 alcohol-related deaths in the UK (Office for National Statistics, UK 2012) and in 2010/11, there were 1,168,300 hospital admissions in England, twice as many as the 510,700 in 2002/03. Some authorities claim that there may be as many as 40,000 people dying every year from alcohol related issues in the UK (Department of Health, 2001). Collating risk factors which lead to alcohol addiction and all its related complications is important for public health (Cobbs, 1992). With substance abuse disorders there is a complex interaction between environmental and genetic factors. With alcoholism there is an interaction between parental influences, genetic susceptibility and social opportunity. Antisocial personality and family influences may bring out the alcoholic trait in genetically predisposed individuals especially women (Lewis \& Bucholz, 1991). Hereditary and genetic factors play a major role in alcoholism (Mayfield et al., 2008). Environmental influences, although important may be overshadowed by a genetic predisposition to alcoholism (Gorwood, 2000). The role of genes in illicit drug abuse is still poorly understood and is a matter of on-going research (Hou \& Li, 2009). Drug seeking behaviour results from developmental and social factors including divorce, childhood neglect, early school leavers and insecurity (Ridenour et al., 2006; Terdi \& Gerevich, 2005). Recent studies related to methamphetamine abuse have shown that people who engaged in 'risky behaviours' were more likely to abuse methamphetamines (Russell et al., 2008). Such behaviours formed a constellation of personality traits which predisposed certain individuals to illicit drug abuse.

Poly drug abuse is common (Senay, 1991). The UNCOPE Study which was done on an arrestee population in the United States showed that almost $65 \%$ of those studied were using multiple substances including alcohol (Hoffmann et al., 2003). Drug abusers commonly experiment with various substances as their habit develops over time. The ease of availability of a specific drug is a determining factor in levels of abuse (Griffiths et al., 2008).

The purpose of poly drug use may be to increase the intoxicating and euphoric experience. One drug may be used to counter the withdrawal effects of another. Cocaine may be used to alleviate the severity of opiate withdrawal (Leri et al., 2003) and benzodiazepines are also used to allay anxiety in many withdrawal syndromes. Such associations might also be expected to occur in the case of alcohol as well (Ndetei et al., 2010). Do alcohol abusers use illicit drugs as part of their intoxicating cocktail?

\section{Aims and Objectives}

The aim of this study was to examine the relationship between alcohol dependency and illicit drug abuse in police detainees. The presence of a significant association between the two could link these two disorders in terms of severity of abuse (Quercioli et al., 2010). The contrary would suggest that the abuse of drugs and alcohol may not be connected and their causations may be different.

\section{Methodology}

Data from 50 successive subjects who had given a history of heavy alcohol consumption to a single clinical forensic medicine examiner were collected across police custodies in West Yorkshire, England, as part of a Clinical Forensic Medical assessment in 2005/06. The participants were: 38 white Caucasians males of West European origin; 9 white Caucasian females of West European origin; 3 males of South Asian origin. All had been arrested by the Police in the investigation of crimes which included 13 allegations of assault, 14 allegations of "disorder", 22 allegations of "theft and related crime" and in one person the charges were "undecided". The medical history included specific enquiry 1) about drinking habits with regard to the amount of alcohol drunk on a daily basis; 2) the type of alcohol beverage which was used regularly and when the subject had his/her last drink; 3) questions regarding tolerance to the effects of alcohol, onset of withdrawal symptoms and excessive drinking on a regular and long term basis (over 1 year) were discussed in detail with all participants. The biological parameters which were checked were based on the 10 common signs and symptoms which form part of the CIWA-Ar (Clinical Institute Withdrawal Assessment) grading scale (Sullivan et al., 1989) for alcohol withdrawal. These were 1) nausea and vomiting 2) tremor 3) paroxysmal sweats 4) anxiety 5) agitation 6) tactile disturbances 7) auditory disturbances 8) visual disturbances 9) headaches 10) orientation and clouding of sensorium. Using the scale points allocated to these signs and symptoms, the numbers were summed. An increasing score indicates a more severe withdrawal syndrome with the maximum score which could be attained on the scale being 67. A baseline pulse and blood pressure, not part of CIWA-Ar scoring, was recorded for all participants. Alcohol addiction category was determined by the level of medical care, which was clinically necessary 
during their detention as dictated by the CIWA-Ar grading system. The principles of CIWA-Ar grading were used for assessment and management of alcohol withdrawal symptoms in all participants.

The UK Misuse of Drugs Act 1971 classifies substances into Class A, B and C based on their harmfulness to the user and to society. For the purpose of this study, each subject's use of illicit drugs was categorised as Non-Class A or Class A drug user to reflect the "street classification" of the drugs used by the participants. "Hard" drugs to refer to heroin, methadone, cocaine, crack cocaine and injectable amphetamine (Class A drugs) and "soft" drugs implied cannabis (Class B and C).

Detailed enquiry about drinking habits showed that the following three DSM-4 Criteria were present in all the participants for more than a year. These were 1) tolerance to alcohol 2) drinking more than intended and 3) the onset of alcohol withdrawal symptoms due to abstinence study.

Abstinence from alcohol for 6 - 8 hours may lead to withdrawal symptoms and deterioration in their general medical condition. All subjects were specifically assessed at 8 hours after their last alcoholic drink. This time interval was chosen to allow the development of alcohol and drug related withdrawal symptoms.

Participants were divided into 3 alcohol groups (See Table 1).

- Group 1 (A1) comprised of 23 participants who did not require any medical intervention for their alcohol withdrawal during their custodial stay. This corresponded to a score of 0 - 8 on the CIWA-Ar scale;

- Group 2 (A2) comprised of 19 participants who had to be given medicines to treat their alcohol withdrawal symptoms during their stay in Police detention. This corresponded to a score of 9 - 20 on the CIWA-Ar scale;

- Group 3 (A3) consisted of 8 participants whose alcohol withdrawal symptoms were so severe that hospital referral was required. This corresponded to a score of above 21 on the CIWA-Ar scale.

The medical history revealed seventeen participants abused illicit drugs; 10 used Class A drugs. Of these, 6 used heroin and methadone, 3 used heroin and crack cocaine and 1 participant used heroin and injectable amphetamine. All heroin users classed themselves as dependent. The accuracy of this opinion was consistent with the DSM-4 criteria for opiate withdrawal. These criteria state that opiate withdrawal occurs in individuals in whom cessation or reduction of heavy opiate use for several weeks or longer leads within minutes or days to any three of these conditions: 1) dysphoric mood; 2) nausea and vomiting; 3) muscle aches; 4) lacrimation or rhinorrhoea; 5) pupillary dilation, piloerection or sweating; 6) diarrhoea; 7) yawning; 8) fever; 9) insomnia. These symptoms cause clinically significant distress or impairment in social, occupational or other important areas of functioning and the symptoms are not due to a general medical condition or another mental health condition.

In the Non-Class A category there were 7 participants and all used cannabis. Each classed their cannabis consumption as misuse and not dependency. Again for the purposes of this study all the participants were divided into 3 drug usage groups.

- The first group (D1) consisted of 33 participants who did not use any illicit drugs;

- The second group (D2) comprised 7 participants who used cannabis Non-Class A drugs;

- The third group (D3) consisted of 10 participants who used Class A drugs.

\section{Ethics}

- This research has been conducted in accordance with research governance and ethical regulations which were adhered to at all times. Ethical approval was obtained for this study from the University of Ulster. All information used in this study was anonymised so that no personal identification occurred regarding the identities of the participants who were included in this study.

Table 1. Summary of participant categories.

\begin{tabular}{cc}
\hline Participant Categories & Number \\
\hline No alcohol withdrawal intervention & 33 \\
Medication for alcohol withdrawal & 23 \\
Alcohol and Cannabis & 19 \\
Severe alcohol withdrawal & 7 \\
Class A drug users & 8 \\
\hline
\end{tabular}

16 of 17 illicit drug users were male. 1 female used Class A drug. 


\section{Data Analysis}

A power calculation was performed to estimate the appropriate size of the sample size for the purposes of this study.

\section{Sample Size Estimation}

To calculate the required sample size the formula $\mathrm{N}>\left(\mathrm{Z}_{\alpha}+\mathrm{Z}_{\beta}\right)^{2} *\left(\frac{\sigma}{\delta}\right)^{2}$ was used where $\alpha$ is the risk of rejecting true positives, $\beta$ is the risk of accepting a false null hypothesis, $\sigma$ is the standard deviation, which is assumed to be known, and $\delta$ is the shift we want to detect. Allowing $\alpha=0.05, \beta=0.1$ and $\delta=0.5 * \sigma$ requires a minimum of 43 samples to keep the risk of detecting false results to a reasonable minimum. In this study, a total of 50 samples were collected.

Data were collected, tabulated and divided into rank tables as above. Descriptive statistics was performed on the data and the Spearman's rank correlation coefficient was calculated to assess the relationship between the severity of abuse of drugs and alcohol dependency. This coefficient gave an accurate estimate of the degree of correlation which existed between alcohol dependency and concomitant drug abuse in the population studied. Kruskal's Gamma is used on original data and was calculated for the series in order to look for any link between the alcohol and drug groups.

\section{Results}

\subsection{Pattern of Offending: Forensic Behavioural Marker}

Twenty seven (54\%) participants had been detained by the Police due to "assaults and disorder" often associated with alcohol dependence. Seven of the ten (70\%) who were abusing Class A drugs had a predilection for "organised crime and theft".

\subsection{Degree of Correlation}

The degree of correlation between alcohol dependency and concomitant drug abuse was tested by calculating the Spearman’s Rank coefficient (Table 2). There was no significant statistical correlation between alcohol dependency and concomitant drug abuse for the series of participants used for the study $p=0.230$.

The Kruskal's Gamma was also calculated which allows us to compare ordinal data and we found no significant link between alcohol group and drug group with $p=0.185$ (See Table 3 ). This suggests that alcohol dependency is distinct from drug abuse and the reliance on one substance does not necessarily imply a reliance on the other.

\subsection{Patterns of Substance Abuse}

This study failed to demonstrate any significant statistical correlation between alcohol dependency and illicit drug co-use. However, a detailed analysis of our data revealed an interesting trend in the pattern of substance abuse. All participants were divided into 5 clusters (Figure 1). Severe drug users were on one side of the scale and a gradation towards the other side of the scale was made based on their increasing severity of alcohol related problems. Cluster 1 had maximum number of participants who used Class A drugs (i.e. D3). In this cluster, there were a total of 10 participants out of which 7 had moderately severe alcohol dependency problems (i.e. A2). The next cluster was of participants who used only Non-Class A drugs (D2). This cluster had 4 participants who had minimal alcohol dependency problems (A1). Cluster 3 had no drugs (D1) and cluster 4 (moderate alcohol dependency problems (A2). In cluster 5 all 7 participants had severe alcohol dependency problems (A3) but only 1 participant used Non-Class A drugs (D2). None of the participants in cluster 5 used Class A drugs. On the drug side of the scale as the degree of addiction rose a mixed picture of misuse emerged e.g. in the cluster which represented severe drug use (D3) there was a considerable overlap with alcohol co-use. In contrast, in the severe alcohol dependency A3 group, there was minimal illicit drug use with only one of 7 subjects in this cluster using only Non-Class A drugs. 
Table 2. Spearman's Correlation Calculations.

\begin{tabular}{ccccc}
\hline & & & Alcohol Group & Drug Group \\
\hline & Alcohol & Correlation Coefficient & 1.0 & 0.173 \\
& group & Sig. (2-tailed) & $\cdot$ & 0.230 \\
Spearman's Correlation & & $\mathrm{N}$ & 50 & 50 \\
Coefficient & Drug & Correlation Coefficient & 0.173 & 1.0 \\
& group & Sig. (2-tailed) & 0.230 & $\cdot$ \\
& & $\mathrm{N}$ & 50 & 50 \\
\hline
\end{tabular}

Spearman’s Correlation Calculations: The correlation between drug and alcohol group is not significant.

Table 3. Kruskal’s gamma calculations.

\begin{tabular}{|c|c|c|c|c|}
\hline & Value & Asymp. Std. Error & Approx. $T^{b}$ & Approx. Sig. \\
\hline Gamma & .270 & .195 & 1.322 & .186 \\
\hline No of Valid Cases & 50 & & & \\
\hline
\end{tabular}

This test is designed for ordinal data and again the connection between the drug and alcohol groups is shown not to be significant.

\begin{tabular}{|l|}
\hline A1-Minimal alcohol dependency problems \\
A2---Moderate alcohol dependency problems \\
A3---Severe alcohol dependency problems \\
D1---No illicit drug abuse \\
D2--Non- Class A drug abuse \\
D3---Class A drug abuse \\
\hline
\end{tabular}

Figure 1. Patterns of substance abuse.

\section{Discussion}

This study failed to show any significant statistical significance between alcohol dependency and illicit drug abuse. It was anticipated that if the study showed any significant correlation between alcohol dependency and concomitant illicit drug abuse, then this might signal the strength of addiction to one substance was mirrored in the other and perhaps common risk factors leading to the genesis of these two disorders. Out of the 50 participants who participated in this study, 66\% did not abuse any illicit drug leaving seventeen (34\%) who were abusing illicit drugs.

Cluster analysis showed a spectrum of abuse with drugs and alcohol dependency of varying degrees. As the severity of drug abuse increased, so did the extent of alcohol related problems in all the relevant clusters. However, the scale moved towards increasing alcohol dependency, correlation with drug co-use diminished and finally disappeared. This is an important finding in this study. The main inference which can be drawn from this study is that alcohol dependency is a unique disorder that is unrelated to other drug addictions.

All addictive drug users go through a cycle of abuse and withdrawal which compels them into an ever increasing cycle of abuse (Gonzales-Saiz et al., 2009). In extreme cases the addict loses contact with reality and is driven by a single minded desire to obtain a constant supply of drugs.

Violent crime was most characteristic of alcohol abuse while in those using Class A drugs on a regular basis, 7 were noted for their involvement in "organised crime". Organised crime is very different from violent disorder as its shows a planned approach towards commission of crime for material benefit. While violent disorder may be a trait associated with alcohol, it is important to remember that all participants were alcohol dependent. Therefore this trait might be expected from all participants. However, Class A drug use changed the behaviour of our study group and led to a predilection for "organised crime". A study in Netherlands found a clear association between substance abuse and criminal behaviour and a distinct link between alcohol use and general violence (Kraanen et al., 2012). A Swedish population study found association between alcohol use and repetitive violence (Bohman et al., 1982). These findings are consistent with our data. However, our study has also highlighted "organised crime" as the forensic behaviour which is linked with Class A misuse. 
Other studies suggest a significant overlap between alcoholism and illicit drug abuse (Barnwell \& Earleywine, 2006; Poikolainen, 1997). In China methadone maintenance patients had a prevalence of multiple drug co-use of 80\% which included cocaine, alcohol and amphetamines (Li et al., 2010). Using the ASSIST, AUDIT and DUDIT questionnaires there is evidence of overlap between the use of alcohol and drugs worldwide (Kader et al., 2012).

Why does this study show no significant correlation between alcohol dependency and illicit drug co-use? Differences in the study population may be responsible-our groups were recruited in a police station whereas recruitment in similar studies was from the community. Custodial recruits belong to a category of people who are at a higher risk of substance abuse disorders (French et al., 2010). The sample size could be insufficient for statistical correlation to emerge but power calculations performed for the statistical model of this study demonstrated that the sample size was adequate for this study but other studies. In addition, is it possible that the medical histories obtained from our custody population are unreliable? However, the latter is unlikely as our findings were corroborated by physical medical evidence in each participant. All the data obtained were anonymised to remove observer bias. The one common characteristic present in all our participants was alcohol dependence. We have clearly shown that there are gradations of association between alcohol and drugs co-use. In Class A drug users there was a strong correlation between alcohol and drugs. However, as the degree of alcohol dependency increased, consumption of other drugs disappeared. This indicates that alcohol dependency is a distinct disorder.

\section{Authors Statement}

The authors can confirm that the material has not been published elsewhere and the paper is not being considered for publication elsewhere. All authors have been personally and substantially involved in the work leading to this paper, and hold ourselves jointly and individually responsible for its content. All authors acknowledge this work as their own. Ethic approval has been granted by the University of Ulster's Ethics Filter Committee. No sources of funding were used for this project. There is no conflict of interest between any of the authors.

\section{References}

Barnwell, S. S., \& Earleywine, M. (2006). Simultaneous Alcohol and Cannabis Expectancies Predict Simultaneous Use. Substance Abuse Treatment, Prevention, and Policy, 11, 1-29.

Bohman, M., Cloninger, C. R., Sigvardsson, S., \&von Knorring, A. L. (1982). Predisposition to Petty Criminality in Swedish Adoptees. 1. Genetic and Environmental Heterogeneity. Archives of General Psychiatry, 39, 1233-1241. http://dx.doi.org/10.1001/archpsyc.1982.04290110001001

Cobbs, L. W. (1992). Shakes and Flashes in a Relapsed Alcoholic. Hospital Practice, 27, 102, 107, 110.

French, M. T., Fang, H., \& Fretz, R. (2010). Economic Evaluation of a Pre-Release Substance Abuse Treatment Program for Repeat Criminal Offenders. Journal of Substance Abuse Treatment, 38, 31-41.

http://dx.doi.org/10.1016/j.jsat.2009.06.001

Gonzales-Saiz, F., Domingo-Salvany, A., Barrio, G., Sanchez-Niubo, A., Brugal, M. T., de la Fuente, L., \& Alonso, J. (2009). Severity of Dependence Scale as a Diagnostic Tool for Heroin and Cocaine Dependence. European Addiction Research, 15, 87-93. http://dx.doi.org/10.1159/000189787

Gorwood, P. (2000). Contribution of Genetics to the Risk Status for Alcohol Dependence. Journal de la Société de Biologie, 194, 43-49.

Griffiths, P., Mravcik, V., Lopez, D., \& Klempova, D. (2008). Quite a Lot of Smoke but Very Limited Fire-The Use of Methamphetamine in Europe. Drug and Alcohol Review, 27, 236-242. http://dx.doi.org/10.1080/09595230801932588

Hoffmann, N. G., Hunt, D. E., Rhodes, W. M., \& Riley, J. (2003). UNCOPE: A Brief Substance Dependence Screen for Use with Arrestees. Journal of Drug Issues, 33, 29-44. http://dx.doi.org/10.1177/002204260303300102

Hou, Q. F., \& Li, S. B. (2009). Potential Association of DRD2 and DAT1 Genetic Variation with Heroin Dependence. Neuroscience Letters, 464, 127-130. http://dx.doi.org/10.1016/j.neulet.2009.08.004

Kader, R., Seedat, S., Koch, J.R., \& Parry, C. D. (2012). A Preliminary Investigation of AUDIT and DUDIT in Comparison to Biomarkers for Alcohol and Drug Use among HIV-Infected Clinic Attendees in Cape Town, South Africa. African Journal of Psychiatry, 15, 346-351. http://dx.doi.org/10.4314/ajpsy.v15i5.43

Kraanen, F. L., Scholing, A., \& Emmelkamp, P. M. (2012). Substance Use Disorders in Forensic Psychiatry: Differences among Different Types of Offenders. International Journal of Offender Therapy and Comparative Criminology, 56, 
1201-1219. http://dx.doi.org/10.1177/0306624X11420252

Leri, F., Bruneau, J., \& Stewart, J. (2003). Understanding Polydrug Use: Review of Heroin and Cocaine Co-Use. Addiction, 98, 7-22. http://dx.doi.org/10.1046/j.1360-0443.2003.00236.x

Lewis, C. E., \& Bucholz, K. K. (1991). Alcoholism, Antisocial Behaviour and Family History. British Journal of Addiction, 86, 177-194. http://dx.doi.org/10.1111/j.1360-0443.1991.tb01768.x

Li, L., Sangthong, R., Chonqsuvivatwong, V., McNeil, E., \& Li, J. (2010). Lifetime Multiple Substance Use Pattern among Heroin Users before Entering Methadone Maintenance Treatment Clinic in Yunnan, China. Drug and Alcohol Review, 29, 420-425. http://dx.doi.org/10.1111/j.1465-3362.2009.00168.x

Mayfield, R. D., Harris, R. A., \& Schuckit, M. A. (2008). Genetic Factors Influencing Alcohol Dependence. British Journal of Pharmacology, 154, 275-287. http://dx.doi.org/10.1038/bjp.2008.88

Ndetei, D. M., Khasakhala, L. I., Mutiso, V., Ongecha-Owuor, F. A., \& Kokonya, D. A. (2010). Drug Use in a Secondary School in Kenya. Journal of Substance Abuse, 31, 170-173. http://dx.doi.org/10.1080/08897077.2010.495313

Poikolainen, K. (1997). Risk Factors for Alcohol Dependence: A Questionnaire Survey. Alcoholism: Clinical and Experimental Research, 21, 957-961.

Quercioli, C., Messina, G., Fini, P., Frola, C., \& Nante, N. (2010). Is It Possible to Evaluate Addiction from Clinical Records? Testing a Retrospective Addiction Severity Evaluation Measure. Substance Use \& Misuse, 45, 2045-2058. http://dx.doi.org/10.3109/10826081003682826

Ridenour, T. A., Maldonado-Molina, M., Compton, W. M., Spitznagel, E. L., \& Cottler, L. B. (2006). Factors Associated with the Transition from Abuse to Dependence among Substance Abusers: Implications for a Measure of Addictive Liability. Drug and Alcohol Dependence, 80, 1-14. http://dx.doi.org/10.1016/j.drugalcdep.2005.02.005

Russell, K., Dryden, D. M., Liang, Y., Friesen, C., O’Gorman, K., Durec, T., Wild, T. C., \& Klassen, T. P. (2008). Risk Factors for Methamphetamine Use in Youth: A Systematic Review. BMC Pediatric, 28, 8-48.

Senay, E. C. (1991). Drug Abuse and Public Health. A Global Perspective. Drug Safety, 1, 1-65.

Sullivan, J. T., Sykora, K., Schneiderman, J., Naranjo, C. A., \& Sellers, E. M. (1989). Assessment of Alcohol Withdrawal: The Revised Clinical Institute Withdrawal Assessment for Alcohol Scale (CIWA-Ar). Br J of Add, 84, 1373-1357.

Terdi, P., \& Gerevich, J. (2005). Substance Use among Homeless and Family Deprived Adolescents. Psychiatr Hung, 20, 348-356. 